\title{
Interval Type-2 Intuitionistic Fuzzy Logic Systems - A Comparative Evaluation
}

\author{
Imo Eyoh ${ }^{1[0000-0002-6548-7644]}$, Robert John ${ }^{10000-0002-2341-9993]}$, and \\ Geert De Maere 2 [0000-0003-0544-1959] \\ 1 Automated Scheduling, Optimisation and Planning (ASAP) and Laboratory for \\ Uncertainty in Data and Decision Making Research Groups, University of \\ Nottingham, UK. \\ $\{i j e, r i j\} @ c s . n o t t . a c . u k$ \\ 2 Automated Scheduling, Optimisation and Planning (ASAP) Research Group, \\ University of Nottingham, UK. \\ gdm@cs.nott.ac.uk
}

\begin{abstract}
Several fuzzy modeling techniques have been employed for handling uncertainties in data. This study presents a comparative evaluation of a new class of interval type-2 fuzzy logic system (IT2FLS) namely: interval type-2 intuitionistic fuzzy logic system (IT2IFLS) of Takagi-Sugeno-Kang (TSK)-type with classical IT2FLS and its type-1 variant (IFLS). Simulations are conducted using a real-world gas compression system (GCS) dataset. Study shows that the performance of the proposed framework with membership functions (MFs) and nonmembership functions (NMFs) that are each intervals is superior to classical IT2FLS with only MFs (upper and lower) and IFLS with MFs and NMFs that are not intervals in this problem domain.
\end{abstract}

Keywords: Interval Type-2 Intuitionistic Fuzzy Logic systems, membership functions, non-membership functions, Decoupled extended Kalman filter

\section{Introduction}

A fuzzy set (FS) is a concept introduced by Zadeh [1] where elements are not restricted to binary MFs of 0 or 1 but rather is a continuum in 0 and 1 . However due to the complexity and uncertainty in many applications, the ordinary FS cannot handle or minimise the uncertainty in many applications because the MF values are exactly defined. To cope with this problem, Zadeh [2] introduced type-2 FS (T2FS) where the MFs are fuzzy with the actual membership grade of an element assumed to lie within a closed interval of 0 and 1. Generally, the definition of classical FSs, both type-1 and type-2 employ only the MFs to define the two concepts: MF and NMF of an element to a set. For classical FSs, the NMF (complement) is 1 minus the membership grade. This definition may not really be the case in real life scenario as they may be some hesitation in the definition of membership degree of an element to a set such that the NMF is not 
complementary to the MF and vice-versa.

Atanassov [3] introduced the concept of intuitionistic FS (IFS) with MF and NMF separately defined such that none is complementary to the other. In the literature, IFSs have been found to be one of the useful tools for dealing with imprecise information [4]. The reader is referred to [5] for more details on IFS. Similar to classical type-1 FSs, the MFs and NMFs of IFSs may not handle the plethora of uncertainty that fraught many applications. The T2FS introduced by Zadeh is a three dimensional structure which provides the extra degrees of freedom needed to handle higher forms of uncertainty. For the generalised T2FS, the third dimension is weighted differently which makes it complex and difficult to use $[6,7]$. The simpler and manageable version - interval type-2 FS (IT2FS) have values in the third dimension equal to 1 and this makes it easier for IT2FS to be represented on a two dimensional plane. The IFSs and IT2FSs have been widely and extensively adopted by researchers in uncertainty modeling in many applications. For example, in Nguyen et al. [8], IT2 fuzzy C-mean (FCM) clustering using IFS is proposed. The authors show that the use of IFS with IT2FCM led to improved clustering quality particularly in the presence of noise. In Naim and Hagras [9, 10] and Naim et al. [11], IFS and IT2FS are combined to develop a multi-criteria group decision making (MCGDM) system for the assessment of post-graduate study, selection of appropriate lighting level in intelligent environment and evaluation of different techniques for the choice of illumination in a shared environments respectively. The authors pointed out that the use of IFS and IT2FS in a MCGDM system provided decisions that are closer to the group decisions compared to some existing methods. However, in $[9,10,11]$, only the IT2FS MFs are utilised and the intuitionistic fuzzy (IF) indices are evaluated on the primary MFs of the IT2FSs and no learning whatsoever is carried out on these sets.

Recently, in Eyoh et al. [12], IFS is fused with IT2FS and an interval type2 intuitionistic FS (IT2IFS) is obtained with artificial neural network learning capability. The uncertainties in IT2IFLS that utilises IT2IFS in the IF-THEN rules are captured by the footprints of uncertainties (FOUs) of both MFs and NMFs. The developed model in [12] is applied for non-linear system prediction with encouraging results. The same authors in Eyoh et al. [13, 14] applied the IT2IFLS framework for time series prediction. Results reveal that IT2IFLS exhibits superior performance to many non-fuzzy and some fuzzy approaches. The authors believe that the additional parameters provided by the NMFs give IT2IFS more design degrees of freedom thus allowing it to minimise the effects of uncertainties in many applications than the classical IT2FLS and IFLS.

Different learning methodologies have been proposed for the adaptation of the parameters of fuzzy logic systems. The study reported here utilises the DEKF to optimise the parameters of the models under investigation. The rest of the paper is structured as follows: In Section 2, the definitions of IFS, T2IFS and IT2IFS are given. The IT2IFLS-TSK model is formulated in Section 3 and parameter update rules are derived in Section 4. We present our experimental set-up and statistical evaluation and discussion in Sections 5 and 6 respectively, and con- 
clude in Section 7. Following are the definitions of some concepts underpinning the proposed model.

\subsection{Intuitionistic Fuzzy Set (IFS)}

Definition 1. For any given finite set $X$, an IFS has the form: $\left.A^{*}=\left\{\left(x, \mu_{A^{*}}(x), \nu_{A^{*}}(x)\right): x \in X\right)\right\}$. where $\mu_{A^{*}}(x): X \rightarrow[0,1]$ is the degree of belonging of $x$ in $X$ and $\nu_{A^{*}}(x): X \rightarrow[0,1]$ is the degree of non-belonging of $x \in X$ with $0 \leq \mu_{A^{*}}(x)+\nu_{A^{*}}(x) \leq 1[3]$.

The IF-index, $\pi_{A^{*}}(x)=1-\left(\mu_{A^{*}}(x)+\nu_{A^{*}}(x)\right)$.

\subsection{Type-2 Intuitionistic Fuzzy Set (T2IFS)}

Definition 2. A T2IFS is of the form: $\tilde{A}^{*}=\left\{(x, u), \mu_{\tilde{A^{*}}}(x, u), \nu_{\tilde{A}^{*}}(x, u) \mid \forall x \in X\right.$, $\left.\forall u \in J_{x}^{\mu}, \forall u \in J_{x}^{\nu}\right\}$ where $\mu_{\tilde{A}^{*}}(x, u)$ is the degree of belonging and $\nu_{\tilde{A}^{*}}(x, u)$ is the degree of non-belonging [12].

$$
\begin{aligned}
J_{x}^{\mu} & =\left\{(x, u): u \in\left[\underline{\mu}_{\tilde{A}^{*}}(x), \bar{\mu}_{\tilde{A}^{*}}(x)\right]\right\} . \\
J_{x}^{\nu} & =\left\{(x, u): u \in\left[\underline{\nu}_{\tilde{A}^{*}}(x), \bar{\nu}_{\tilde{A}^{*}}(x)\right]\right\} .
\end{aligned}
$$

in which $0 \leq \mu_{\tilde{A}^{*}}(x, u) \leq 1$ and $0 \leq \nu_{\tilde{A}^{*}}(x, u) \leq 1, J_{x}^{\mu}$ and $J_{x}^{\nu}$ represent the support of the secondary MFs and NMFs on the third dimension [12].

The T2IFS $\tilde{A}^{*}$ can also be formulated as:

$$
\tilde{A}^{*}=\int_{x \in X}\left[\int_{u \in J_{x}^{\mu}} \int_{u \in J_{x}^{\nu}}\left\{\mu_{\tilde{A}^{*}}(x, u), \nu_{\tilde{A}^{*}}(x, u)\right\}\right] /(x, u) .
$$

for continuous universe of discourse (UoD). For discrete UoD, $\tilde{A}^{*}$ becomes

$$
\tilde{A}^{*}=\sum_{x \in X}\left[\sum_{u \in J_{x}^{\mu}} \sum_{u \in J_{x}^{\nu}}\left\{\mu_{\tilde{A}^{*}}(x, u), \nu_{\tilde{A}^{*}}(x, u)\right\}\right] /(x, u) .
$$

When $\mu_{\tilde{A}^{*}}(x, u)=1$, and $\nu_{\tilde{A}^{*}}(x, u)=1$, an interval type-2 intuitionistic FS (IT2IFS) is obtained. The IF-indices utilised are defined as follows [12]:

$$
\begin{gathered}
\pi_{c}(x)=\max \left(0,\left(1-\left(\mu_{\tilde{A}^{*}}(x)+\nu_{\tilde{A}^{*}}(x)\right)\right)\right) . \\
\bar{\pi}_{v a r}(x)=\max \left(0,\left(1-\left(\bar{\mu}_{\tilde{A}^{*}}(x)+\underline{\nu}_{\tilde{A}^{*}}(x)\right)\right)\right) . \\
\underline{\pi}_{v a r}(x)=\max \left(0,\left(1-\left(\underline{\mu}_{\tilde{A}^{*}}(x)+\bar{\nu}_{\tilde{A}^{*}}(x)\right)\right)\right) .
\end{gathered}
$$

Two footprints of uncertainties (FOUs) defined for IT2IFS are as follows [12]:

$$
\begin{aligned}
& \operatorname{FOU}_{\mu}\left(\tilde{A}^{*}\right)=\bigcup_{\forall x \in X}\left[\underline{\mu}_{\tilde{A}^{*}}(x), \bar{\mu}_{\tilde{A}^{*}}(x)\right] . \\
& \operatorname{FOU}_{\nu}\left(\tilde{A}^{*}\right)=\bigcup_{\forall x \in X}\left[\underline{\nu}_{\tilde{A}^{*}}(x), \bar{\nu}_{\tilde{A}^{*}}(x)\right] .
\end{aligned}
$$

denoting MF and NMF FOUs respectively. 


\section{Interval Type-2 Intuitionistic Fuzzy Logic System}

The structure of T2FLS rule based system is exactly as that of the classical T2FLS with components comprising of the intuitionistic fuzzifier, intuitionistic rule base, intuitionistic inference engine and intuitionistic output processing module.

\section{$2.1 \quad$ Fuzzification}

The fuzzification process maps the crisp input vector $x \in X$ into an IT2IFS $\tilde{A}^{*}$ thereby assigning to each element its MF and NMF degree in each IT2IFS partition.

The IT2IFS is defined using a modified Gaussian function (see Fig. 1 and (10) to (13)) [12]. The scaling and shifting allows for the degree of indeterminacy to be embedded in the FOUs of IT2IFLS.

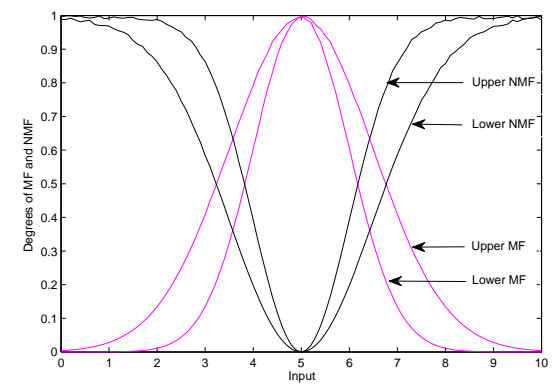

Fig. 1. A typical IT2IFS [12]

$$
\begin{gathered}
\overline{\mu_{i k}}\left(x_{i}\right)=\exp \left(-\frac{\left(x_{i}-c_{i k}\right)^{2}}{2 \bar{\sigma}_{2, i k}^{2}}\right) *\left(1-\pi_{c, i k}\left(x_{i}\right)\right) . \\
\underline{\mu_{i k}}\left(x_{i}\right)=\exp \left(-\frac{\left(x_{i}-c_{i k}\right)^{2}}{2 \underline{\sigma}_{1, i k}^{2}}\right) *\left(1-\pi_{c, i k}\left(x_{i}\right)\right) . \\
\overline{\nu_{i k}}\left(x_{i}\right)=\left(1-\bar{\pi}_{v a r, i k}\left(x_{i}\right)\right)-\left[\exp \left(-\frac{\left(x_{i}-c_{i k}\right)^{2}}{2 \bar{\sigma}_{1, i k}^{2}}\right) *\left(1-\pi_{c, i k}\left(x_{i}\right)\right)\right] . \\
\underline{\nu_{i k}}\left(x_{i}\right)=\left(1-\underline{\pi}_{v a r, i k}\left(x_{i}\right)\right)-\left[\exp \left(-\frac{\left(x_{i}-c_{i k}\right)^{2}}{2 \underline{\sigma}_{2, i k}^{2}}\right) *\left(1-\pi_{c, i k}\left(x_{i}\right)\right)\right] .
\end{gathered}
$$

where $\pi_{c, i k}$ is the IF-index of center and $\pi_{v a r, i k}$ is the IF-index of variance [4]. 


\section{$2.2 \quad$ Rules}

The IF-THEN rule formation of IT2IFLS follows the general fuzzy logic rule syntax. For IT2IFLS-TSK, the antecedent are IT2IFSs while the consequent parts are linear combinations of the inputs. A typical rule structure of IT2IFLS is as shown in (14).

$$
R_{k}: \operatorname{IF} x_{1} \text { is } \tilde{A}^{*}{ }_{1 k} \text { and } \cdots \text { and } x_{n} \text { is } \tilde{A}^{*}{ }_{n k} \text { THEN } y_{k}=\sum_{i=1}^{n} w_{i k} x_{i}+b_{k} .
$$

For IT2IFLS, the rules are defined for MFs in (15) and NMFs in (16) as follows:

$$
\begin{aligned}
& R_{k}^{\mu}: \operatorname{IF} x_{1} \text { is } \tilde{A}^{*}{ }_{1 k}^{\mu} \text { and } \cdots \text { and } x_{n} \text { is } \tilde{A}^{*}{ }_{n k}^{\mu} \text { THEN } y_{k}^{\mu}=\sum_{i=1}^{n} w_{i k}^{\mu} x_{i}+b_{k}^{\mu} . \\
& R_{k}^{\nu}: \operatorname{IF} x_{1} \text { is } \tilde{A}^{*}{ }_{1 k}^{\nu} \text { and } \cdots \text { and } x_{n} \text { is } \tilde{A}^{*}{ }_{n k}^{\nu} \text { THEN } y_{k}^{\nu}=\sum_{i=1}^{n} w_{i k}^{\nu} x_{i}+b_{k}^{\nu} .
\end{aligned}
$$

where $\tilde{A}^{*}{ }_{1 k}, \tilde{A}^{*}{ }_{2 k}, \cdots, \tilde{A}^{*}{ }_{i k}, \cdots, \tilde{A}^{*}{ }_{n k}$ are IT2IFS, $y_{k}^{\mu}$ and $y_{k}^{\nu}$ are the MFs and NMFs outputs of the $k t h$ rule, $w^{\prime}$ s and $b$ 's are consequent parameters.

\subsection{Inference}

This study adopts a TSK-inferencing system where the inputs are IT2IFS and the output of each IF-THEN rule is a linear function of the inputs, otherwise known as A2-C0 TSK-fuzzy inferencing. The inference engine of IT2IFLS is defined in $(17)[12,13]$.

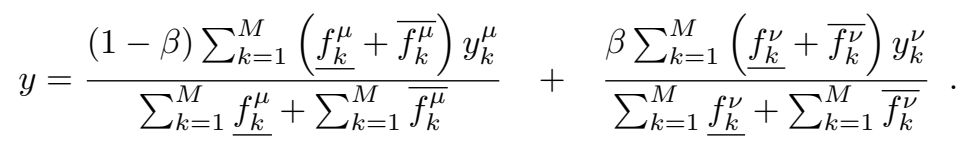

and utilises the "prod" t-norm to specify the firing strength such that:

$$
\begin{gathered}
\underline{f_{k}^{\mu}}(x)=\prod_{i=1}^{n} \underline{\mu}_{\tilde{A}^{*}{ }_{i k}}\left(x_{i}\right), \quad \overline{f_{k}^{\mu}}(x)=\prod_{i=1}^{n} \bar{\mu}_{\tilde{A}^{*}{ }^{*}}\left(x_{i}\right) . \\
\underline{f_{k}^{\nu}}(x)=\prod_{i=1}^{n} \underline{\nu}_{\tilde{A}^{*}{ }_{i k}}\left(x_{i}\right), \quad \overline{f_{k}^{\nu}}(x)=\prod_{i=1}^{n} \bar{\nu}_{\tilde{A}^{*}{ }_{i k}}\left(x_{i}\right) .
\end{gathered}
$$

where $\underline{f}_{k}$ and $\bar{f}_{k}$ are the lower and upper firing strengths defined for both MFs and NMFs respectively, $y_{k}^{\mu}$ and $y_{k}^{\nu}$ are the outputs of the $k t h$ rule corresponding to MF and NMF respectively. The final output of IT2IFLS-TSK is a weighted average of each IF-THEN rule's output. The parameter $\beta(0 \leq \beta \leq 1)$ weighs the contribution of the MF and NMF values in the final output. 


\section{Parameter Update}

In this section, the parameter update rules for IT2IFLS using DEKF is derived. For comparison, the DEKF is also used to update the parameters of classical IT2FLS and IFLS.

\subsection{Extended Kalman filter Parameter Update Rule}

The purpose of IT2IFLS prediction is to obtain an accurate an estimate as possible between input-output relationship of a system. Let the output of IT2IFLS be $y=f(X, \theta)$. The parameter $X$ denotes the inputs into the system and $\theta$ is used to represent the unknown parameters of the model. The generic state equation of the non-linear system can be expressed as:

$$
\begin{gathered}
\theta_{t+1}=f\left(\theta_{t}\right)+\omega_{t} . \\
y_{t}=h\left(\theta_{t}\right)+v_{t} .
\end{gathered}
$$

where $\theta$ is the system's state, $\omega$ is the process noise with zero mean and covariance $Q$ and $v$ is the measurement noise with zero mean and covariance $R$. The process and measurement noise are assumed to be Gaussian and uncorrelated and:

$$
\begin{array}{r}
E\left(\theta_{0}\right)=\bar{\theta}_{0}, \quad E\left[\left(\theta_{0}-\bar{\theta}_{0}\right)\left(\theta_{0}-\bar{\theta}_{0}\right)^{T}\right]=P_{0}, \\
E\left(\omega_{t}\right)=0, \quad E\left(\omega_{t} \omega_{l}^{T}\right)=Q \delta_{t l}, \\
E\left(v_{t}\right)=0, \quad E\left(v_{t} v_{l}^{T}\right)=R \delta_{t l} .
\end{array}
$$

where $E($.$) is the expectation operator and \delta_{t l}$ is the Kronecker delta. Using Taylor expansion, the state is estimated as:

$$
\begin{aligned}
& f\left(\theta_{t}\right)=f\left(\hat{\theta}_{t}\right)+F_{t}\left(\theta_{t}-\hat{\theta}_{t}\right)+\text { H.O.T. } \\
& h\left(\theta_{t}\right)=h\left(\hat{\theta}_{t}\right)+H_{t}\left(\theta_{t}-\hat{\theta}_{t}\right)+H . O . T .
\end{aligned}
$$

where:

$$
F_{t}=\left.\frac{\partial f(\theta)}{\partial \theta}\right|_{\theta=\hat{\theta}_{t}} \quad \text { and } \quad H_{t}^{T}=\left.\frac{\partial h(\theta)}{\partial \theta}\right|_{\theta=\hat{\theta_{t}}} .
$$

and H.O.T is the higher order term. The system in (23) can be approximated as in (24) when the H.O.Ts are neglected.

$$
\begin{aligned}
& \theta_{t+1}=F_{t} \theta_{t}+\omega_{t}+\phi_{t} . \\
& y_{t+1}=H_{t}^{T} \theta_{t}+v_{t}+\varphi_{t} .
\end{aligned}
$$

where:

$$
\begin{aligned}
\phi_{t} & =f\left(\hat{\theta}_{t}\right)-F_{t} \hat{\theta}_{t} . \\
\varphi_{t} & =h\left(\hat{\theta}_{t}\right)-H_{t} \hat{\theta}_{t} .
\end{aligned}
$$


The order of the computational cost of EKF is $A B^{2}$, where $A$ is the output dimension of the system and $B$ is the number of parameters. An IT2IFLS with $n$ inputs and $M$ rules has a total of $6 n+2 M(n+1)$ parameters. The computational cost of EKF for IT2IFLS is $36 n^{2}+4 M^{2}\left(n^{2}+2 n+1\right)+24 n M(n+1)$.

\subsection{Decoupled Extended Kalman Filter - DEKF}

In using the DEKF to learn the parameters of IT2IFLS, the antecedent and the consequent parameters for both MFs and NMFs are grouped into two vectors namely: antecedent $\left(\theta^{1}\right)$ and consequent $\left(\theta^{2}\right)$ parameter vectors. The generic parameter update rules for the parameters in the $i t h$ group is expressed in (25) to $(27)$ :

$$
\begin{gathered}
\theta_{t}^{i}=\theta_{t-1}^{i}+K_{t}^{i}\left[y_{t}-h\left(\theta_{t-1}\right)\right] \\
K_{t}^{i}=P_{t}^{i} H_{t}^{i}\left[\left(H_{t}^{i}\right)^{T} P_{t}^{i} H_{t}^{i}+R^{i}\right]^{-1} \\
P_{t+1}^{i}=P_{t}^{i}-K_{t}^{i} P_{t}^{i}\left(H_{t}^{i}\right)^{T}+Q^{i}
\end{gathered}
$$

where $K$ is the Kalman gain, $P$ is the covariance matrix of the state estimation error. The unknown parameters in the antecedent are gathered into the antecedent's parameter vector and represented as (28):

$$
\theta^{1}=\left[c_{11}, c_{21}, \cdots, c_{n N}, \sigma_{11}, \sigma_{21}, \cdots, \sigma_{n N}\right]^{T} .
$$

where $n$ is the number of inputs and $N$ is the number of linguistic terms. The parameters of the consequent are grouped into the consequent's parameter vector and represented as (29):

$$
\theta^{2}=\left[w_{11}, w_{21}, \cdots, w_{M n}, b_{1}, b_{2}, \cdots, b_{M}\right]^{T} .
$$

where $M$ is the number of rules, with the MF and NMFs having separate Kalman parameters. The derivative matrix, $\mathrm{H}$, is defined as:

$$
H^{1}=\frac{\partial y}{\partial \theta^{1}} \quad \text { and } \quad H^{2}=\frac{\partial y}{\partial \theta^{2}}
$$

for antecedent and consequent parameters respectively. The update rule for the antecedent and consequent parameters then follow the same recursive procedures in $(25)$ to (27). Using the DEKF algorithm reduces the computational burden of EKF in the order $36 n^{2}+4 M^{2}\left(n^{2}+2 n+1\right)$ such that the computational cost of DEKF to standard EKF is in the ratio:

$$
\frac{36 n^{2}+4 M^{2}\left(n^{2}+2 n+1\right)}{36 n^{2}+4 M^{2}\left(n^{2}+2 n+1\right)+24 n M(n+1)}
$$


In the next sections, the IT2IFLS is used for the prediction of a real-world dataset - GCS dataset of a gas turbine obtained from a Nigerian-based power plant. The purpose of this simulation is to statistically analyse the performance of IT2IFLS with other existing FLSs such as the classical IT2FLS and the type-1 IFLS. The DEKF learning approach is adopted for these experimental analyses because of its theoretical strength, faster convergence and its ability at finding good solutions [15].

\section{Experimental Set-up}

The GCS data is a complex dataset consisting of different operational conditions of a gas plant. The GCS data consist of 825 data points and modeled as a time series using input generating format: $[y(t-3), y(t-2), y(t-1)]$ with $y(t)$ as the output. The inputs are normalised to lie between small range of $[0,1]$, so that larger input values do not overshadow the smaller values, thereby leading to poor prediction and learning using the embedded neural network architecture. For each run of the experiments, the data are randomly sampled and split into $70 \%$ training and 30\% testing set with each data point having equal chance of being chosen for training and testing. For a clear and objective discussion and evaluation of the three models of IT2IFLS, IT2FLS and IFLS, the Kalman filter parameters $R, Q$ and $P$ for both MFs and NMFs are initially set as $40,0.01 I_{32}$ and $1.0 I_{32}$ respectively for all experiments with 100 epochs for each run. The simulation is conducted for 30 runs. This allows for objective evaluation of the performance of the different models. Figures 2 and 3 show a single GCS input partition before and after training using IT2IFLS.
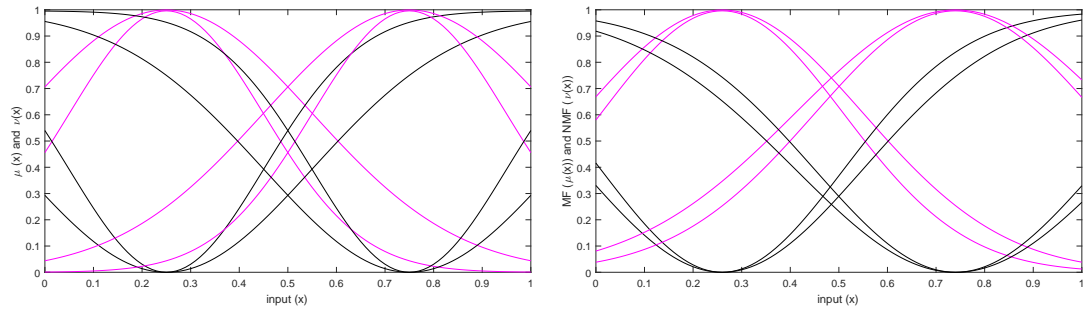

Fig. 2. GCS $x_{1}$ before training with Fig. 3. GCS $x_{1}$ after training with IT2IFLS-DEKF

IT2IFLS-DEKF

\section{Statistical Evaluation and Discussion}

In this section, statistical evaluation is conducted to test the hypothesis of this research. The main interest is to understand the effectiveness of integrating NMF 
and IF-indices into the classical IT2FLS (IT2IFLS). The second is to investigate the performance of the proposed framework of IT2IFLS with its type-1 counterpart. Statistical comparison is also made between IFLS and IT2FLS. To explore these analyses, three experiments are conducted. In each case, the performance metric is the root mean squared error (RMSE). Figure 1 shows the box-plot of the three models using their test RMSEs. The following hypotheses form the basis of evaluation:

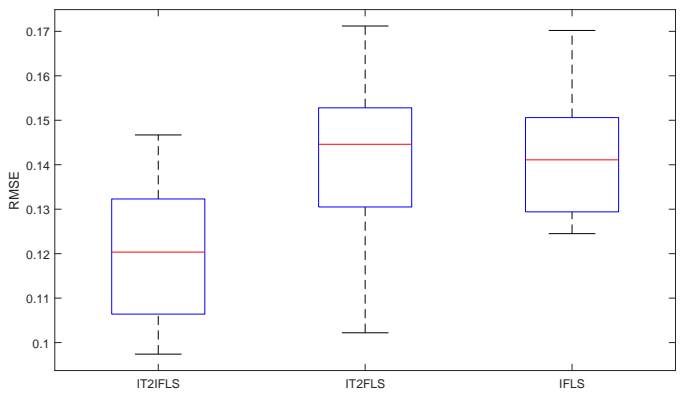

Fig. 4. Box and whisker plot showing the performance of IT2IFLS, IT2FLS and IFLS trained with DEKF.

- Hypothesis 1: With the integration of NMFs and IF-indices into the classical IT2FLS, the new model of IT2IFLS is able to model uncertainty in many applications than the classical IT2FLS that do not incorporate NMFs and IF-indices.

- Hypothesis 2: With MFs and NMFs that are intervals, the new model of IT2IFLS is able to model uncertainty in many applications than its type-1 variant with MFs and NMFs that are not represented as intervals values.

- Hypothesis 3: With MFs and NMFs of IFLS, the model is able to model uncertainty in many applications than the classical IT2FLS with only interval MFs (lower and upper).

Statistical significant differences (one-tailed) between pairs of models are carried out using Wilcoxon signed rank test $(\alpha$ level $=0.05)$, which is a nonparametric statistical hypothesis test for evaluating pairs of models. The test RMSEs averaged over 30 runs for the different fuzzy models considered here are presented in Table 1.

Hypothesis 1: The first set of experiments is focused on assessing the ability of IT2IFLS framework to provide good estimates than the classical IT2FLS. The null and the alternative hypotheses are:

- $H_{0}$ : There is no significant difference in the uncertainty modeling employing IT2FLS that incorporates NMFs and IF-indices and those that do not. 
Table 1. Gas Compression System Prediction

\begin{tabular}{|c|c|c|}
\hline Models & Training RMSE & Test RMSE \\
\hline IT2FLS-TSK & 0.1504 & 0.1425 \\
\hline IFLS-TSK & 0.1496 & 0.1423 \\
\hline IT2IFLS-TSK & 0.1202 & 0.1199 \\
\hline
\end{tabular}

- $H_{1}$ : There is a significant difference in the uncertainty modeling of IT2FLS that incorporates NMFs and IF-indices and those that do not.

Table 2. Wilcoxon's test: IT2IFLS vs IT2FLS on test data RMSE

\begin{tabular}{lcc}
\hline Models & Hypothesis $(\alpha=0.05)$ & $p$-value \\
\hline IT2IFLS vs IT2FLS & Reject $H_{0}$ & 0.0173 \\
\hline
\end{tabular}

For the first hypothesis, the statistical analysis in Table 2 shows that there is a significant difference between the uncertainty modeling using the two FLSs $(p$-value $=0.0173)$. Based on this premise, the null hypothesis is rejected with a conclusion that there is a significant difference between the performance of IT2IFLS compared to the classical IT2FLS. As observed in the box-and-whisker plot, IT2IFLS has a smaller error value on average. This observation demonstrates the advantages of NMFs and IF-indices as an integral part of IT2FLS (IT2IFLS).

Hypothesis 2: The second set of experiments is focused on assessing the ability of IT2IFLS framework to provide good estimates than its type-1 counterpart. The null and the alternative hypotheses are:

- $H_{0}$ : There is no significant difference in uncertainty modeling of IT2IFLS with MFs and NMFs that are each intervals and IFLS with MFs and NMFs that are not intervals.

- $H_{1}$ : There is a significant difference in uncertainty modeling of IT2IFLS with MFs and NMFs that are each intervals and IFLS with MFs and NMFs that are not intervals.

Table 3. Wilcoxon's test: IT2IFLS vs IFLS on test data RMSE

\begin{tabular}{lcc}
\hline Models & Hypothesis $(\alpha=0.05)$ & $p$-value \\
\hline IT2IFLS vs IFLS & Reject $H_{0}$ & 0.0091 \\
\hline
\end{tabular}

For the second hypothesis, the statistical analysis in Table 3 suggests that there is a significant difference between the uncertainty modeling using IT2IFLS com- 
pared to IFLS ( $p$-value $=0.0091)$. With this $p$-value, the null hypothesis is rejected. It is concluded that there is a significant difference between the IT2IFLS and IFLS. This shows that IT2IFLSs with MFs and NMFs that are intervals may be more appropriate for uncertainty modeling than those with MFs and NMFs representations that are not intervals.

Hypothesis 3: The third set of experiments is to investigate the statistical significance between IT2FLS and IFLS. The null and the alternative hypotheses are:

- $H_{0}$ : There is no significant difference in the performance of IT2FLS utilising upper and lower MFs of IT2FS and IFLS utilising MFs and NMFs.

$-H_{1}$ : There is a significant difference in the performance of IT2FLS utilising upper and lower MFs of IT2FS and IFLS utilising MFs and NMFs.

Table 4. Wilcoxon's test: IT2FLS vs IFLS using test data RMSE

\begin{tabular}{lrr}
\hline Models & Hypothesis $(\alpha=0.05)$ & $p$-value \\
\hline IT2FLS vs IFLS & Fail to reject $H_{0}$ & 0.7336 \\
\hline
\end{tabular}

Table 4 shows the results of statistical comparison between classical IT2FLS and type-1 IFLS. The Wilcoxon's signed rank test at 0.05 significance level shows that there is no significant difference $(p$-value $=0.7336)$ existing between classical IT2FLS and IFLS, therefore, we fail to reject the null hypothesis.

\section{Conclusion}

In this study, the DEKF is used to optimise the parameters of IT2IFLS, classical IT2FLS and type-1 IFLS. Specifically, the following conclusions are supported:

- IT2IFLS captures more information and enables hesitation in the FS description.

- There is significant performance improvements of IT2IFLS over IT2FLS and IFLS.

- The performance of the classical IT2FLS is comparable to that of IFLS.

- The IT2IFLS with MF and NMF that are intervals can minimise the effects of uncertainties in most applications.

In the future, we intend to conduct more experiments using bench mark data sets, other fuzzy modeling functions such as triangular and trapezoidal functions and other learning algorithms.

\section{Acknowledgement}

This research work was supported by the Government of Nigeria under the Tertiary Education Trust Fund (TETFund). 


\section{References}

[1] Zadeh, L.A.: Fuzzy sets. Information and control 8(3) (1965) 338-353

[2] Zadeh, L.A.: The concept of a linguistic variable and its application to approximate reasoning-i. Information Sciences 8 (1975) 199-249

[3] Atanassov, K.T.: Intuitionistic fuzzy sets. Fuzzy sets and Systems 20(1) (1986) $87-96$

[4] Hájek, P., Olej, V.: Intuitionistic fuzzy neural network: The case of credit scoring using text information. In: Engineering Applications of Neural Networks. Springer (2015) 337-346

[5] Atanassov, K.T.: On Intuitionistic Fuzzy Sets Theory. Springer-Verlag (2012)

[6] Mendel, J.M., John, R.I., Liu, F.: Interval type-2 fuzzy logic systems made simple. IEEE Transactions on Fuzzy Systems 14(6) (2006) 808-821

[7] Mendel, J.M., John, R.B.: Type-2 fuzzy sets made simple. Fuzzy Systems, IEEE Transactions on 10(2) (2002) 117-127

[8] Nguyen, D.D., Ngo, L.T., Pham, L.T.: Interval type-2 fuzzy c-means clustering using intuitionistic fuzzy sets. In: IEEE Third World Congress on Information and Communication Technologies (WICT). (2013) 299-304

[9] Naim, S., Hagras, H.: A hybrid approach for multi-criteria group decision making based on interval type-2 fuzzy logic and intuitionistic fuzzy evaluation. In: Fuzzy Systems (FUZZ-IEEE), 2012 IEEE International Conference on, IEEE (2012) 1-8

[10] Naim, S., Hagras, H.: A type 2-hesitation fuzzy logic based multi-criteria group decision making system for intelligent shared environments. Soft Computing 18(7) (2014) 1305-1319

[11] Naim, S., Hagras, H., Bilgin, A.: Employing an interval type-2 fuzzy logic and hesitation index in a multi criteria group decision making system for lighting level selection in an intelligent environment. In: Advances in Type-2 Fuzzy Logic Systems (T2FUZZ), 2013 IEEE Symposium on, IEEE (2013) 1-8

[12] Eyoh, I., John, R., De Maere, G.: Interval type-2 intuitionistic fuzzy logic system for non-linear system prediction. In: Systems, Man, and Cybernetics (SMC), 2016 IEEE International Conference on, IEEE (2016) 001063-001068

[13] Eyoh, I., John, R., Maere, G.D.: Time series forecasting with interval type-2 intuitionistic fuzzy logic systems. In: 2017 IEEE International Conference on Fuzzy Systems (FUZZ-IEEE). (July 2017) 1-6

[14] Eyoh, I., John, R., De Maere, G.: Extended kalman filter-based learning of interval type-2 intuitionistic fuzzy logic system. In: 2017 IEEE International Conference on Systems, Man and Cybernetics. (2017) 728-733

[15] Simon, D.: Training fuzzy systems with the extended kalman filter. Fuzzy sets and systems 132(2) (2002) 189-199 